\title{
Primitive neuroectodermal tumor originating from the lung: A case report
}

\author{
XIN JIN $^{1}$, JIANFENG CAO ${ }^{2}$, YONG LIU ${ }^{1}$, FANG BIAN ${ }^{1}$, QINGQING ZHAO ${ }^{1}$, \\ YAN WANG $^{1}, \mathrm{XU} \mathrm{LV}^{3}$ and YAYONG HUANG ${ }^{4}$ \\ ${ }^{1}$ Department of Oncology; ${ }^{2}$ Intensive Care Unit; Departments of ${ }^{3}$ Pathology and ${ }^{4}$ Radiology, \\ Xuzhou Central Hospital, Xuzhou, Jiangsu 221009, P.R. China
}

Received April 2, 2015; Accepted May 17, 2016

DOI: $10.3892 / \mathrm{ol} .2016 .4973$

\begin{abstract}
Primitive neuroectodermal tumors (PNETs) are small, round cell tumors that may be classified as peripheral or central, based on their site of origin. PNETs often arise in the soft tissue or bone of young adults. Although not common, PNETs have been described in other organs, including the gonads, kidneys, myocardium and pancreas, but rarely in the lungs without chest wall or pleural involvement. The present study reports a rare case of peripheral PNET (pPNET), which originated in the lung. A 37-year-old female patient presented at Xuzhou Central Hospital (Xuzhou, China) with a history of a dry cough, mild dyspnea and slight pain in the left chest. Histopathological and immunohistochemical analyses permitted the diagnosis of a pPNET. The patient was treated with surgical resection, followed by chemotherapy (including cyclophosphamide, cisplatin and vincristine), radiotherapy and traditional Chinese medicine (including Kanglaite and Shenqi Fuzheng injections). At the time of writing, the patient was alive with no sign of recurrence and under regular follow-ups at the Outpatient Clinic of Xuzhou Central Hospital.
\end{abstract}

\section{Introduction}

Primitive neuroectodermal tumors (PNETs) are rare, undifferentiated sarcomas deriving from cells that originate from the neural crest. PNETs often arise in the soft-tissues and bones of adolescents and young adults (aged $<35$ years), with a slight male preponderance (1). Primary peripheral PNET (pPNET) of the lung without chest wall or pleural involvement is extremely rare. Due to the similarities of PNET and Ewin's tumor, it is difficult to estimate the exact incidence of PNET. The most common symptoms are a cough, fever,

Correspondence to: Mr. Yong Liu, Department of Oncology, Xuzhou Central Hospital, 199 South Jiefang Road, Xuzhou, Jiangsu 221009, P.R. China

E-mail: lyly_oncology@126.com

Key words: primitive neuroectodermal tumor, lung, diagnosis, treatment dyspnea, hemoptysis and chest pain; however, none of the clinical manifestations are specific to PNET (2). The diagnosis of PNET is generally made by a histopathological analysis, and the treatment of PNET involves various combinations of surgical resection, chemotherapy and radiotherapy (3). Similar to Ewing's sarcoma, PNET is a highly malignant tumor with a poor prognosis: the 5-year survival rate is $<25 \%$ (4).

The present study reports the rare case of a patient with pPNET localized to the lung, who was successfully treated by a combination of surgical resection, radiotherapy (RT), chemotherapy and traditional Chinese medicine, including Kanglaite and Shenqi Fuzheng injections.

\section{Case report}

In March 2013, a 37-year-old female patient presented at the Department of Thoracic Surgery of Xuzhou Central Hospital (Xuzhou, China), with a history of a dry cough, mild dyspnea and slight pain in the left chest for 3 months. A computed tomography (CT) scan of the chest revealed a mass with lobulated margins (Fig. 1A). The mass was not connected to the chest walls, pleurae or any other adjacent organs (Fig. 1B). Bronchoscopy did not reveal any abnormal results. Standard staging procedures, including bone emission $\mathrm{CT}$, brain magnetic resonance imaging and abdominal ultrasonography, identified no distant metastasis. The values of the serum tumor markers neuron-specific enolase (NSE), carcinoembryonic antigen, squamous cell carcinoma and cytokeratin 19 fragments were not elevated.

A left upper lobectomy associated with mediastinal lymph node dissection was performed in the Department of Thoracic Surgery in April 2013, and neither the pleurae or the chest wall were involved. Microscopic analysis indicated that the resected tissue consisted of small round cells (Fig. 1C). Immunohistochemical analysis of 4- $\mu \mathrm{m}$ formalin-fixed, paraffin-embedded tissue sections stained with hematoxylin and eosin and visualized using the MaxVision TM HRP-Polymer antiMouse/Rabbit IHC kit (cat. no. KIT-5030; Fuzhou Maixin Biotech., Co., Ltd., Fuzhou, China) demonstrated that the tumor cells were positive for cluster of differentiation (CD) 99 (mouse anti-CD99 monoclonal antibody; cat. no. MAB-0059) (Fig. 1D) and CD56 (mouse anti-CD56 monoclonal antibody; cat. no. MAB-0256) (Fig. 1E), focally positive for 
A

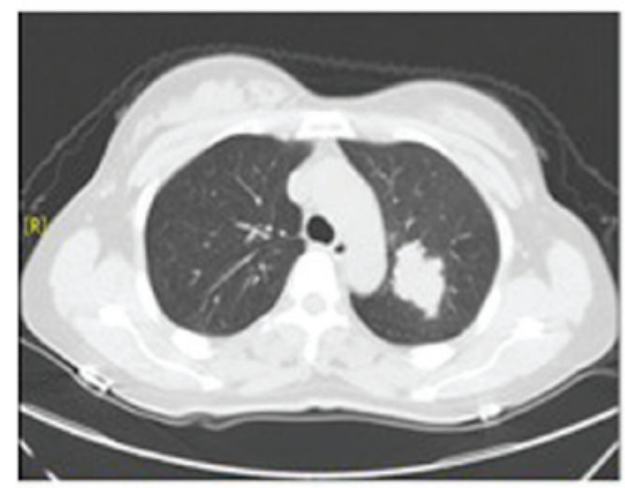

C

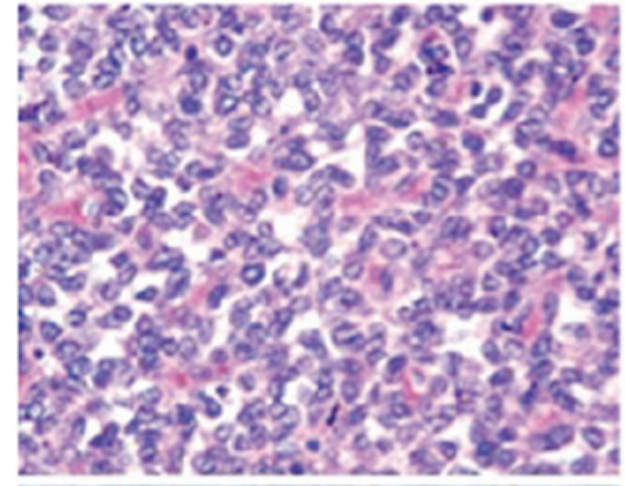

$\mathbf{E}$

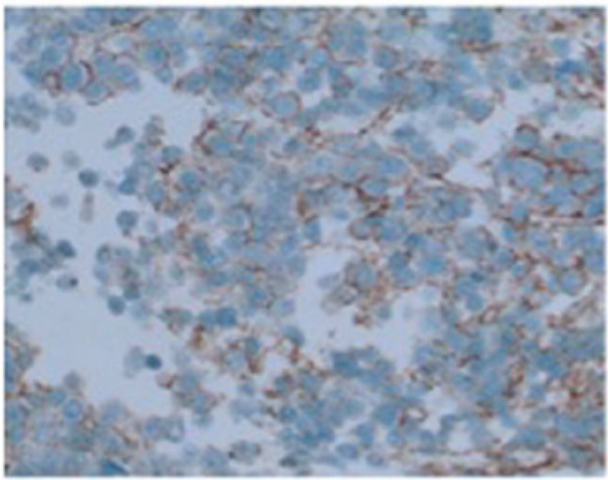

B

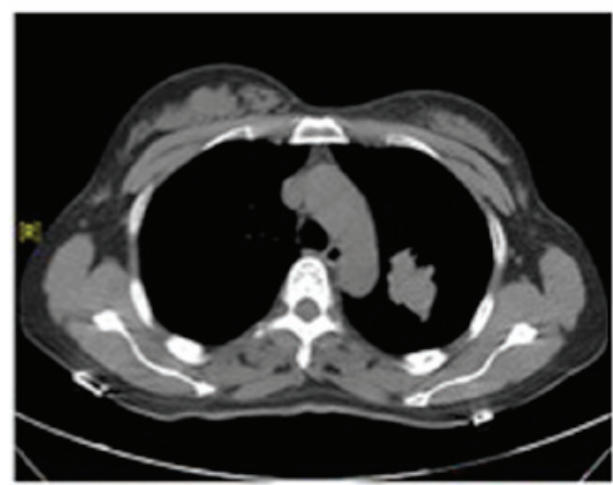

D

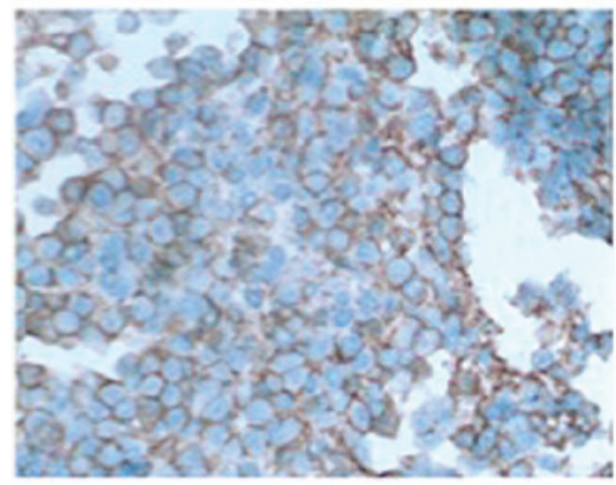

$\mathbf{F}$

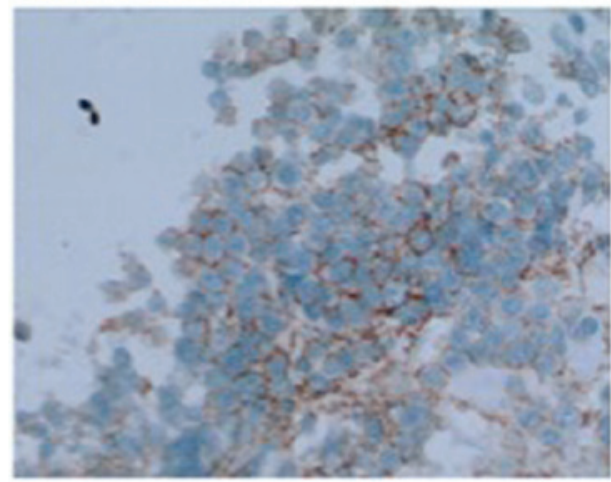

Figure 1. Computed tomography scan of the chest showing (A) a mass with lobulated margins that (B) was not connected to the pleurae, chest wall or other adjacent organs. (C) Hematoxylin and eosin staining showing that the tumor was composed of small, round-to-oval nuclei (magnification, $x 400$ ). (D-F) Immunohistochemical staining showing that the tumor cells were positive for (D) CD99 and (E) CD56, and were (F) focally positive for vimentin (magnification, $\mathrm{x} 400$ ). $\mathrm{CD}$, cluster of differentiation.

vimentin (rabbit anti-vimentin monoclonal antibody; cat. no. RMA-0547) (Fig. 1F), and negative for thyroid transcription factor 1 (TTF-1; mouse anti-TTF-1 monoclonal antibody; cat. no. MAB-0266), S-100 (mouse anti-S-100 monoclonal antibody; cat. no. MAB-0697), leukocyte common antigen (LCA; mouse anti-CD45 monoclonal antibody; cat. no. MAB-0037) and chromogranin A (mouse anti-chromogranin A; cat. no. MAB-0202; all: Fuzhou Maixin Biotech., Co., Ltd.). These findings were indicative of a pPNET, and the diagnosis was supported by the presence of the $\mathrm{t}(11 ; 22)(\mathrm{q} 24 ; \mathrm{q} 21)$ translocation in the tumor cells, as detected by a cytogenetic analysis.

Following the diagnosis of pPNET, the patient initially received RT. The total RT dose was $50 \mathrm{~Gy}$, which was administered with a daily standard fractionation schedule of $2 \mathrm{~Gy}$. Following RT, the patient underwent a combined chemotherapy regime with cyclophosphamide $\left(750 \mathrm{mg} / \mathrm{m}^{2}\right)$, cisplatin $\left(75 \mathrm{mg} / \mathrm{m}^{2}\right)$ and vincristine $\left(1.4 \mathrm{mg} / \mathrm{m}^{2}\right)$ every 3 weeks for a total of 6 cycles. Following completion of chemotherapy, a chest $\mathrm{CT}$ scan was performed, which showed no disease progression. Traditional Chinese medicine, including Kanglaite (200 ml/day for 21 days; Zhejiang Kanglaite Pharmaceutical Co., Ltd., Zhejiang, China) and Shenqi Fuzheng $(250 \mathrm{ml} /$ day for 10 days; Livzon Pharmaceutical Group, Inc., Guangdong, China) injections, were administered monthly until the present in order to improve the patient's immune functions and decrease adverse events associated with chemotherapy and radiotherapy. Follow-ups were scheduled every 3 months, and the patient is currently in a good condition. Written informed consent was obtained from the patient for the publication of this study.

\section{Discussion}

pPNETs belong to the family of 'small round cell tumors', which exhibit various degrees of neuroectodermal differentiation and derive from cells originating from the neural crest (5). This rare neoplasm is more frequent in children and adolescents than in adults (1). PNETs involving the 
thoracopulmonary region were first reported as 'malignant small cell tumors of the thoracopulmonary region in childhood' by Askin et al in 1979, which resulted in them being termed as Askin's tumors (6).

Although pPNETs have often been reported in the literature, the majority are located in the kidneys, chest wall, urinary bladder, myocardium, retroperitoneum, pancreas and the female genital tract (7-9). Reports of pPNETs arising in the lung without chest wall or pleural involvement, such as the one in the present case, are extremely rare.

The diagnosis of pPNETs is based on light microscopy following identification of a small round cell tumor (10). Immunohistochemically, pPNETs are positive for CD99, NSE, CD56 and vimentin, and negative for LCA, cytokeratin, epithelial membrane antigen and desmin (10). In order to diagnose a tumor as a pPNET, it should be positive for at least two of the aforementioned neural markers. In addition, reciprocal translocation $(11 ; 22)(\mathrm{q} 24 ; \mathrm{q} 12)$ is considered to be characteristic of this tumor family $(11,12)$. Any tumor suspected to be a pPNET should undergo biopsy, either by needle or a complete and wide surgical excision in order to obtain tissue from the lesion for all aforementioned tests. Hence, the diagnosis of pPNETs is based on histopathological, immunohistochemical, and, when possible, genetic analyses.

Due to the different therapeutic schedules and prognostic characteristics for distinct tumor types, differential diagnosis is essential for PNETs. Usually, PNETs share a similar histological appearance with small round blue cell tumor (except for the presence of rosettes), and CD99 expression and cytogenetic translocation $\mathrm{t}(11 ; 22)(\mathrm{q} 24 ; \mathrm{q} 12)$ with Ewing's sarcoma. Neural differentiation indicates the presence of PNET rather than Ewing's sarcoma (13). The differential diagnosis of PNET also includes small-cell carcinoma, neuroblastoma, lymphoma and rhabdomyosarcoma, which are all indistinguishable by conventional light microscopy (5). Positive immunohistochemical staining for CD99, CD56, vimentin, NSE and synaptophysin are favorable in the differential diagnosis of PNET (6).

Neuroblastomas are also positive for NSE and synaptophysin, but negative for CD99, and the presence of Homer-Wright rosettes is a characteristic of these lesions (14). LCA positivity supports the diagnosis of lymphoma, but $\mathrm{T}$ cell lymphoblastic lymphoma may be positive for CD99 and CD3, and negative for LCA. Small-cell carcinoma is almost always positive for cytokeratin, while rhabdomyosarcoma is positive for actin, desmin and myoglobin (15-18); therefore, the immunohistochemical results observed in the present case (positivity for CD99, vimentin and CD56, and negativity for CD3, desmin, and LCA) highly support the diagnosis of a pulmonary PNET.

Treatment for pPNETs includes surgical resection, chemotherapy and radiotherapy. It has been reported that complete surgical excision with wide $(2-3 \mathrm{~cm})$ margins may improve long-term survival for patients with PNETs (19). The most commonly recommended chemotherapy regimens include several cycles with agents such as cyclophosphamide, vincristine, doxorubicin, etoposide and ifosfamide $(13,20)$. A number of studies have reported poor long-term survival rates in PNETs despite multimodal treatment $(21,22)$. The patient in the present case was treated by a multimodal treatment strategy that included surgery, radiotherapy and 6 cycles of chemotherapy with cyclophosphamide, cisplatin and vincristine. Furthermore, traditional Chinese medicine, including Kanglaite and Shenqi Fuzheng injections, were used for the treatment. Kanglaite injection is an antitumor agent that has been shown to significantly decrease the occurrence of cancer cachexy and improve the quality of life of cancer patients (23). In addition, it may ameliorate the development of multiple drug resistance in cancers when combined with radiotherapy and chemotherapy, as well as strengthening the overall response rate and reducing the side effects of nausea and vomiting (24). Shenqi Fuzheng injection is commonly used to improve immune function against cancer, and was reported to reduce the toxicity of radiotherapy and chemotherapy (25). At the time of writing, the patient had been alive without any signs of recurrence or metastasis for 19 months, which demonstrated that the treatment had been adequate.

In conclusion, despite the rarity of arising from the lung without chest wall or pleural involvement in adult patients, PNETs should be considered in the differential diagnosis of all parenchymal lung nodules. In addition, multimodal treatment, including surgical excision, radiotherapy, feasible chemotherapy regimens and traditional Chinese medicine, has been shown to be beneficial.

\section{Acknowledgements}

The present study was supported by the project Six Talent Peaks Project of Jiangsu Province (grant no. WSN-065).

\section{References}

1. de Alava E and Gerald WL: Molecular biology of the Ewing's sarcoma/primitive neuroectodermal tumor family. J Clin Oncol 18: 204-213, 2000.

2. Dong M, Liu J, Song Z, Li X, Shi T, Wang D, Ren D and Chen J: Primary Multiple Pulmonary Primitive Neuroectodermal Tumor: Case Report and Literature Review. Medicine (Baltimore) 94: e1136, 2015.

3. Andrei M, Cramer SF, Kramer ZB, Zeidan A and Faltas B: Adult primary pulmonary primitive neuroectodermal tumor: Molecular features and translational opportunities. Cancer Biol Ther 14: 75-80, 2013.

4. Subbiah V, Anderson P, Lazar AJ, Burdett E, Raymond K and Ludwig JA: Ewing's sarcoma: Standard and experimental treatment options. Curr Treat Options Oncol 10: 126-140, 2009.

5. Jo VY and Fletcher CD: WHO classification of soft tissue tumours: An update based on the 2013 (4th) edition. Pathology 46: 95-104, 2014.

6. Askin FB, Rosai J, Sibley RK, Dehner LP and McAlister WH: Malignant small cell tumor of the thoracopulmonary region in childhood: A distinctive clinicopathologic entity of uncertain histogenesis. Cancer 43: 2438-2451, 1979.

7. Khosla D, Rai B, Patel FD, Sreedharanunni S, Dey P and Sharma SC: Primitive neuroectodermal tumor of the uterine cervix diagnosed during pregnancy: A rare case with review of literature. J Obstet Gynaecol Res 40: 878-882, 2014.

8. Kakkar S, Gupta D, Kaur G and Rana V: Primary primitive neuroectodermal tumor of kidney: A rare case report with diagnostic challenge. Indian J Pathol Microbiol 57: 298-300, 2014.

9. Banerjee SS, Eyden BP, McVey RJ, Bryden AA and Clarke NW: Primary peripheral primitive neuroectodermal tumor of the urinary bladder. Histopathology 30: 486-490, 1997.

10. Jimenez RE, Folpe AL, Lapham RL, Ro JY, O'Shea PA, Weiss SW and Amin MB: Primary Ewing's sarcoma/primitive neuroectodermal tumor of the kidney: A clinicopathologic and immunohistochemical analysis of 11 cases. Am J Surg Pathol 26: 320-327, 2002. 
11. Lee YY, Kim do H, Lee JH, Choi JS, In KH, Oh YW, Cho KH and Roh YK: Primary pulmonary Ewing's sarcoma/primitive neuroectodermal tumor in a 67-year-old man. J Korean Med Sci 22 (Suppl): S159-S163, 2007.

12. Kuroda M, Urano M, Abe M, Mizoguchi Y, Horibe Y, Murakami M, Tashiro K and Kasahara M: Primary primitive neuroectodermal tumor of the kidney. Pathol Int 50: 967-972, 2000.

13. Carvajal R and Meyers P: Ewing's sarcoma and primitive neuroectodermal family of tumors. Hematol Oncol Clin North Am 19: 501-525, 2005

14. Small AG, Thwe le M, Byrne JA, Lau L, Chan A, Craig ME, Cowell CT and Garnett SP: Neuroblastoma, body mass index, and survival: A retrospective analysis. Medicine (Baltimore) 94: e713, 2015.

15. Mentzel T, Flaschka J, Mentzel HJ,Eschholz G and Katenkamp D Primary primitive neuroectodermal tumor of the urinary bladder: Clinicopathologic case report and differential small cell tumor diagnosis of this site. Pathologe 19: 154-158, 1998.

16. Shi L, Guo Z and Wu X: Primary pulmonary primitive neuroectodermal tumor metastasis to the pancreas: A rare case with seven-year follow-up. Diagn Pathol 8: 51, 2013.

17. Cetiner H, Kir G, Gelmann EP and Ozdemirli M: Primary vulvar Ewing sarcoma/primitive neuroectodermal tumor: A report of 2 cases and review of the literature. Int J Gynecol Cancer 19 $1131-1136,2009$

18. Colecchia M, Dagrada G, Poliani PL, Messina A and Pilotti S: Primary primitive peripheral neuroectodermal tumor of the prostate: Immunophenotypic and molecular study of a case. Arch Pathol Lab Med 127: e190-e193, 2003.
19. Craver RD, Lipscomb JT, Suskind D and Velez MC: Malignant teratoma of the thyroid with primitive neuroepithelial and mesenchymal sarcomatous components. Ann Diagn Pathol 5: 285-292, 2001

20. Grier HE, Krailo MD, Tarbell NJ,Link MP, Fryer CJ,Pritchard DJ, Gebhardt MC, Dickman PS, Perlman EJ, Meyers PA, et al: Addition of ifosfamide and etoposide to standard chemotherapy for Ewing's sarcoma and primitive neuroectodermal tumor of bone. N Engl J Med 348: 694-701, 2003.

21. Mulsow J, Jeffers M, McDermott R, Geraghty J and Rothwell J: Complete clinical response to neoadjuvant chemotherapy in a 54-year old male with Askin tumor. Thorac Cardiovasc Surg 58: 306-308, 2010

22. Gunluoglu MZ, Kara HV, Demir A and Dincer SI: Results of multimodal treatment of two patients with thoracic primitive neuroectodermal tumor. Is surgery really helpful for survival? Thorac Cardiovasc Surg 55: 460-461, 2007.

23. Qi F, Zhao L, Zhou A, Zhang B, Li A, Wang Z and Han J: The advantages of using traditional Chinese medicine as an adjunctive therapy in the whole course of cancer treatment instead of only terminal stage of cancer. Biosci Trends 9: 16-34, 2015.

24. Wang JC, Tian JH, Ge L, Gan YH and Yang KH: Which is the best Chinese herb injection based on the FOLFOX regimen for gastric cancer? A network meta-analysis of randomized controlled trials. Asian Pac J Cancer Prev 15: 4795-4800, 2014.

25. Li J, Wang JC, Ma B, Gao W, Chen P, Sun R and Yang KH: Shenqi Fuzheng Injection for advanced gastric cancer: A systematic review of randomized controlled trials. Chin J Integr Med 21: 71-79, 2015. 